\title{
ON THE DISTRIBUTION OF THE TIME TO FIRST EMPTINESS OF A STORE WITH STOCHASTIC INPUT
}

\author{
A. M. HASOFER
}

(received 15 April 1964)

\section{Introduction}

Kendall [4] has given for the distribution of the time to first emptiness in a store with an input process which is homogeneous and has non-negative independent increments and an output of one unit per unit time the formula

$$
g(t, z)=\frac{z}{t} k(t, t-z) .
$$

In this formula, $z$ is the initial content of the store, $g(t, z)$ is the density function of the time to first emptiness $\tau(z)$, defined by

$$
P\{\tau(z) \leqq t\}=G(t, z)=\int_{0}^{t} g(u, z) d u,
$$

and $k(t, x)$ is the density function of the input process $\xi(t)$, defined by

$$
P\{\xi(t) \leqq x\}=K(t, x)=\int_{0}^{x} k(t, y) d y .
$$

Lloyd [5] has given the corresponding formula for the case of a discrete input in the form

$$
q_{n}(z)=\frac{z}{z+n} p_{n}(z+n), \quad n=0,1,2, \cdots
$$

where

$$
\begin{aligned}
& q_{n}(z)=P\{\tau(z)=z+n\}, \\
& p_{n}(z)=P\{\xi(t)=n\} .
\end{aligned}
$$

However, as pointed out by Lloyd, Kendall did not establish formula (1) as giving the density function of $\tau(z)$. Kendall only showed that (1) satisfied the integral equation

$$
g(t, z)=\int_{0}^{t-z} g(t-z, y) k(z, y) d y .
$$

In fact, it can be easily shown that the integral equation (2) has the general solution 


$$
g(t, z)=\int_{0-}^{t-z} k(t, t-z-x) d P(x)
$$

where $P(x)$ is an arbitrary function of bounded variation. The particular solution (1) is obtained by taking $P(x)=U(x)-K_{10}(0,2)$, where $K(t, x)=$ $P\{\xi(t) \leqq x\}, K_{10}(0, x)=\partial K(t, x) /\left.\partial t\right|_{t=0}$, and

$$
U(x)= \begin{cases}1 & \text { for } x \geqq 0 \\ 0 & \text { otherwise. }\end{cases}
$$

The last result can be obtained as follows: we must solve the equations

$$
\int_{0-}^{t-z} k(t, t-z-x) d P(x)=\frac{z}{t} k(t, t-z) .
$$

We know that the Laplace transform of $k(t, x)$ can be written in the form

$$
\int_{0}^{\infty} e^{-s x} k(t, x) d x=e^{-\alpha(s) t}
$$

because of the additivity of the process $\xi(t)$.

Writing $t-z=u, P(x)=U(x)-Q(x)$, we find that

$$
\int_{0-}^{u} k(t, u-x) d Q(x)=\frac{u}{t} k(t, u) .
$$

Take Laplace transforms. This yields

$$
e^{-\alpha(s) t} \int_{0}^{\infty} e^{-s x} d Q(x)=\alpha^{\prime}(s) e^{-\alpha(s) t},
$$

i.e. the Laplace-Stieltjes transform of $Q(x)$ is $\alpha^{\prime}(s)$.

But

$$
\alpha^{\prime}(s)=\left.\int_{0}^{\infty} e^{-s x} k_{10}(t, x) d x\right|_{t=0}=\int_{0}^{\infty} e^{-s x} d K_{10}(0, x),
$$

so that we can take $Q(x)$ to be $K_{10}(0, x)$. Our final answer is therefore

$$
P(x)=U(x)-K_{10}(0, x) .
$$

In this paper we shall prove the following under very mild general restrictions:

(a) If the input process has a density function $k(t, x)$, then $\tau(z)$ has a density function $g(t, z)$, and

$$
g(t, z)= \begin{cases}\frac{z}{t} k(t, t-z) & \text { if } t \geqq z \\ 0 & \text { otherwise. }\end{cases}
$$


(b) If $\xi(t)$ is a Compound Poisson process, then the distribution function, $G(t, z)$, of $\tau(z)$, is given by

$$
G(t, z)= \begin{cases}\frac{z}{t} K(t, t-z)-\int_{z}^{t} \frac{z}{u^{2}}\left[u K_{10}(u, u-z)-K(u, u-z)\right] d u \text { if } t \geqq z \\ 0 & \text { otherwise. }\end{cases}
$$

In particular, formula (4) will hold for discrete inputs, and we shall show that it reduces to Lloyd's formula in that case.

\section{Definition and measurability of the time of first emptiness}

We shall consider a store with an infinite capacity, having an input $\xi(t)$ in the time interval $(0, t]$, and a "planned output function" $\eta(t)$ during the same period. By this we mean that if the store did not become empty at any time during the period $(0, t]$, the realised output would be $\eta(t)$. We shall assume that $\xi(t)$ and $\eta(t)$ are arbitrary non-decreasing functions which are continuous to the right and bounded in any finite interval, and such that $\xi(0)=\eta(0)=0$.

We shall set $v(t)=\xi(t)-\eta(t)$. Then $v(t)$ will be the "net planned input" to the store. We shall now further assume that $\nu(t)$ has no downwards discontinuities, i.e. that $v(t)-v(t-) \geqq 0$. Let $v^{*}(t)=-\inf _{0 \leqq u \leqq t} v(u)$. Then $\nu^{*}(t)$ is a non-decreasing function of $t$ with no discontinuities. Let $z$ be the initial content of the store. Kingman [3] has shown that the content, $\zeta(t)$, of the store at time $t$ can be defined by the following formula

$$
\zeta(t)=v(t)+\max \left[z ; v^{*}(t)\right] .
$$

Let now $\tau(z)$ be the time elapsing until the store becomes empty for the first time, i.e. the smallest value of $t$ for which $\zeta(t)=0$. We shall first show that $\tau(z)$ is the smallest value of $t$ for which $v^{*}(t)=z$. In fact if $t_{0}$ is this smallest value, we have $v^{*}\left(t_{0}\right)=-v\left(t_{0}\right)$ and therefore $\zeta(t)=0$. Moreover, as $\nu^{*}(t)$ is non-decreasing, we have, for all $t<t_{0}, \nu^{*}(t)<z$. This implies $-v(t) \leqq \nu^{*}(t)<z$, and consequently $\zeta(t)=\nu(t)+z>0$.

Suppose now that the net planned input is a stochastic process $\nu(t, \omega)$. As $v$ is a function of bounded variation in $t, v(t, \omega)$ is separable. We now show that $\tau(z)$ is a random variable, i.e. a measurable function of $\omega$.

The event $\{\omega ; \tau(z) \leqq t\}$ is given by

$$
\begin{aligned}
\{\omega ; \tau(z) \leqq t\} & =\left\{\omega ; \nu^{*}(t) \geqq z\right\} \\
& =\{\omega ; \inf \nu(u) \leqq-z\} \\
& =\bigcup_{0 \leqq u \leqq t}\{\omega ; \nu(u) \leqq-z\} .
\end{aligned}
$$


It follows that the event $\{\omega ; \tau(z) \leqq t\}$ is measurable, and therefore $\tau(z)$ is a (possibly defective) random variable. We now make the furcher assumption that $v(t)$ is a homogeneous stochastic process with independent increments, i.e. we shall assume that $\delta v=v(t+\delta t)-v(t)$ is a random variable which is independent of $v(t)$ and whose distribution depends on $\delta t$ only. In this case it is well known that we can write the Laplace-Stieltjes transform of the distribution function of $v(t)$ as

$$
E\left[e^{-s \nu(t)}\right]=e^{-\phi(s) t} .
$$

THEOREM 1. Under the above assumptions, the Laplace-Stieltjes transform $\Gamma(p, z)=E[\exp \{-p \tau(z)\}]$ is given by $\Gamma(p, z)=\exp \{-\theta z\}$, where $\theta$ satisfies the equation

$$
p=-\phi(\theta) \text {. }
$$

Proof. Because of the assumptions on the nature of the process $v(t)$, we obviously have

$$
\tau(y+z)=\tau(y)+\tau(z)
$$

where $\tau(y)$ and $\tau(z)$ are independent. It follows that the Laplace-Stieltjes transform of the distribution of $\tau(z), \Gamma(p, z)$, where

$$
\Gamma(p, z)=E\left[e^{-p \tau(z)}\right],
$$

is of the form $\exp \{-\theta z\}$, where $\theta$ is some function of $p$. We also have the relation

$$
\tau[\eta(z)]=z+\tau[\xi(z)]
$$

for if the initial content of the store is $\eta(z)$, after a period of time of length $z$ the initial content has been exhausted, and the new content is the input in the period $[0, z]$, namely $\xi(z)$. We now extend the definition of $\tau(z)$ to negative values of the argument by setting $\tau(-z)=-\tau(z)$. Equation (5) then formally generalises to negative values of $y$ and $z$. Thus we can rewrite (6) as $\tau[\xi(z)-\eta(z)]=-z$ or $\tau[v(z)]=-z$.

From this we deduce

$$
\begin{aligned}
e^{p z}=E[\exp \{-p \tau[v(z)]\}] & =E\{E[\exp \{-p \tau[v(z)]\} \mid v(z)]\} \\
& =E[\exp \{-\theta \nu(z)\}] \\
& =\exp \{-\phi(\theta) z\},
\end{aligned}
$$

so that finally $p=-\phi(\theta)$.

\section{The Lagrange expansion of $\Gamma(p, z)$ in the case of an output of one unit per unit time}

Let us now consider the special case where the input $\xi(t)$ is a homoge- 
neous process with independent increments and the planned output is given by $\eta(t)=t$.

We shall write

$$
E\left[e^{-s \xi(t)}\right]=e^{-\alpha(s) t} .
$$

We shall assume that $\alpha(s)$ can be expressed in the form

$$
\alpha(s)=\int_{0-}^{\infty}\left(e^{-\varepsilon x}-1\right) d M(x),
$$

where $M(x)$ is a non-decreasing function such that $M(\infty)=0$. We shall further assume that $\lim _{x \rightarrow 0} x M(x)=0$. That $\exp \{-\alpha(s) t\}$ then corresponds to some process $\xi(t)$ with independent increments follows from the general theory of infinitely divisible distributions. See, for instance, Gnedenko and Kolmogorov [2].

Integrating by parts, we find

$$
\alpha(s)=s \int_{0}^{\infty} e^{-s x} M(x) d x=s \beta(s), \text { say. }
$$

We shall also assume that $\alpha^{\prime}(0)$ is finite, and consequently, as

$$
\alpha^{\prime}(0)=\lim _{s \rightarrow 0} \alpha(s) \mid s=\lim _{s \rightarrow 0} \int_{0}^{\infty} e^{-s x} M(x) d x,
$$

the last limit will exist.

Finally, we note that in this case, the function which we had previously denoted by $\phi(s)$ is now equal to $\alpha(s)-s$, so that equation (7) becomes

$$
p=\theta-\alpha(\theta) \text {. }
$$

We now introduce the following

ThEOREM 2. There exist two real positive numbers $p_{0}, \sigma_{0}$ such that equation (8) has exactly one root $\theta$ satisfying $\operatorname{Re}(\theta)>\sigma_{0}$, for all real values of $p$ satisfying $p>p_{0}$. Moreover, if $f(z)$ is a function analytic in $\operatorname{Re}(z)>\sigma_{0}$, $f(\theta)$ is given by

$$
f(\theta)=f(p)+\sum_{n=1}^{\infty} \frac{1}{n !} \frac{d^{n-1}}{d p^{n-1}}\left[f^{\prime}(p)\{\alpha(p)\}^{n}\right] .
$$

Proof. Let $s=\sigma+i \omega$. Then $\lim _{\sigma \rightarrow \infty} \beta(\sigma+i \omega)=0$. Moreover, $|\beta(\sigma+i \omega)|$ $\leqq \beta\left(\sigma_{0}\right)$ for all $\sigma \geqq \sigma_{0}$ and all $\omega$. It follows that we can choose $\sigma_{0}$ such that $|\beta(s)| \leqq \mu<\frac{1}{2}$ for all $s$ such that $\operatorname{Re}(s)>\sigma_{0}$. We then have, in the same region, $|\alpha(s)| \leqq \mu|s|$. We now show that, if $p$ is real and $|s-p|>\mu p /(1-\mu)$, we have $|s-p|>|\alpha(s)|$ for all $s$ such that $\operatorname{Re}(s)>\sigma_{0}$. In fact, we then have

$$
|\alpha(s)| \leqq \mu|s|=\mu|s-p+p| \leqq \mu|s-p|+\mu p<|s-p| .
$$


Finally we note that if $p$ satisfies the inequality $p>(1-\mu) \sigma_{0} /(1-2 \mu)$, all points such that $|s-p| \leqq \mu p /(1-\mu)$ will have an abscissa larger than $\sigma_{0}$, so that every point in $\operatorname{Re}(s)>\sigma_{0}$ can be surrounded by a contour $C$ in the same region containing the circle $|s-p|=\mu p /(1-\mu)$. On this contour, we shall have $|s-p|>|\alpha(s)|$, and by applying Rouché's theorem, we conclude that the equation $s-p=\alpha(s)$ has only one root in $\operatorname{Re}(s)>\sigma_{0}$. Moreover, any function $f(z)$ which is analytic in a region containing the contour $C$ can be expanded by using Lagrange's theorem, yielding the expansion given in the theorem.

Corollary.

$$
\Gamma(p, z)=e^{-\theta z}=e^{-p z}-z \sum_{n=1}^{\infty} \frac{1}{n !} \frac{d^{n-1}}{d p^{n-1}}\left[e^{-p z}\{\alpha(p)\}^{n}\right] .
$$

Proof. The only point requiring checking is whether the root $\theta$ in the expression for $\Gamma(p, z)$ is the same as the one discussed in the theorem. This, however follows from the formula $\lim _{p \rightarrow \infty} \Gamma(p, z)=P\{\tau(z)=0\}=0$ for $z>0$, which implies $\lim _{p \rightarrow \infty} \operatorname{Re}(\theta)=+\infty$.

\section{The inversion of $\Gamma(p, z)$ when the input has a density function}

THEOREM 3. Let the distribution of $\xi(t)$ have a density function $k(t, x)$. Moreover, let the Laplace-Stieltjes transform of $\xi(t)$ be of the form $\exp [-\alpha(s) t]$ where $\alpha(s)=s \int_{0}^{\infty} e^{-s x} M(x) d x$ and $\alpha^{\prime}(0)$ is finite. Then $\tau(z)$ has a density function $g(t, x)$, which is given by

provided that

$$
g(t, x)=\frac{z}{t} k(t, t-z) \text { for almost all } t,
$$

$$
\int_{y}^{\infty} e^{-D t} \frac{y}{t} k(t, t-y) d t
$$

is of bounded variation in $y$ in some neighbourhood of $y=z$.

Proof. We have

$$
\int_{0}^{\infty} e^{-s x} k(t, x) d x=e^{-\alpha(s) t}, \quad \operatorname{Re}(s) \geqq 0,
$$

where $\operatorname{Re}[\alpha(s)] \geqq 0$. We deduce that

$$
\int_{0}^{\infty} \int_{0}^{\infty} e^{-p t-s x} k(t, x) d x=\frac{1}{p+\alpha(s)}, \quad \operatorname{Re}(p)>0 .
$$

Let us for the moment restrict $s$ and $p$ to real positive values, and change variables in ( $1 \dot{0})$ by replacing $x$ by $t-z$. We find that 


$$
\frac{1}{p+\alpha(s)}=\int_{0}^{\infty} \int_{-\infty}^{t} e^{-(p+s) t+s z} k(t, t-z) d z d t .
$$

Write now $p$ for $p+s$. We obtain

$$
\frac{1}{p-s+\alpha(s)}=\int_{0}^{\infty} e^{-\downarrow t}\left\{\int_{-\infty}^{t} e^{s z} k(t, t-z) d z\right\} d t .
$$

Differentiate both sides with respect to $s$. We have

$$
\frac{1-\alpha^{\prime}(s)}{[p-s+\alpha(s)]^{2}}=\int_{0}^{\infty} e^{-p t}\left\{\int_{-\infty}^{t} e^{s z} z k(t, t-z) d z\right\} d t,
$$

where the double integral still converges absolutely. obtain

Integrate both sides of (11) with respect to $p$ from $p$ to infinity. We

$$
-\frac{1-\alpha^{\prime}(s)}{p-s+\alpha(s)}=\int_{0}^{\infty} e^{-p t}\left\{\int_{-\infty}^{t} e^{s z} \frac{z}{t} k(t, t-z) d z\right\} d t .
$$

Let us put

$$
g^{*}(t, z)= \begin{cases}\frac{z}{t} k(t, t-z) & \text { for } z \leqq t \\ 0 & \text { otherwise. }\end{cases}
$$

Then we can write (12) as

$$
-\frac{1-\alpha^{\prime}(s)}{p-s+\alpha(s)}=\int_{0}^{\infty} e^{-p t} \int_{-\infty}^{+\infty} e^{s z} g^{*}(t, z) d z d t
$$

As the double integral converges absolutely, we can use Fubini's theorem to interchange the integrals, thus obtaining

$$
-\frac{1-\alpha^{\prime}(s)}{p-s+\alpha(s)}=\int_{-\infty}^{+\infty} e^{s z}\left\{\int_{0}^{\infty} e^{-p t} g^{*}(t, z) d t\right\} d z .
$$

As the integral converges for all positive values of $p$, and all values of $s$ such that $\operatorname{Re}(s)>0$, the last equation holds for all $s$ such that $\operatorname{Re}(s)>0$.

Now

$$
\lim _{R \rightarrow \infty}-\frac{1}{2 \pi i} \int_{c-\imath R}^{c+\imath R} \frac{\left[1-\alpha^{\prime}(s)\right] e^{-s z}}{p-s+\alpha(s)} d s=e^{-\theta z}, \quad z>0,
$$

if $\sigma_{0}<c<\mu p /(1-\mu)$, where $\sigma_{0}$ and $\mu$ are as defined in the theorem of section $3, p>(1-\mu) \sigma_{0} /(1-2 \mu)$, and $\theta$ is the unique root of $p-s+\alpha(s)=0$ in $\operatorname{Re}(s)>\sigma_{0}$. This follows immediately from the fact that in $\operatorname{Re}(s)>\sigma_{0}$, $\left|1-\alpha^{\prime}(s)\right| \leqq 1+\alpha^{\prime}(0)$ and is therefore bounded, and $|p-s+\alpha(s)|>|s|-p$ $-\mu|s|>\frac{1}{2}|s|-p$, so that the integral along the semi-circle of radius $R$ with 
centre at $s=c$ which lies to the right of the line $\operatorname{Re}(s)=c$ tends to zero when $R \rightarrow \infty$. It now follows from a theorem of Widder [7], p. 241, on the bilateral Laplace transform, that

$$
\int_{0}^{\infty} e^{-p t} g^{*}(t, z) d t=e^{-\theta z}, \quad z>0,
$$

for all sufficiently large real positive $p$.

Finally, it follows from the uniqueness theorem for Laplace transform (see Widder $[6]$, p. 63), that

$$
g^{*}(t, z)=g(t, z) \text { for almost all } t .
$$

This completes the proof of the theorem.

\section{The inversion of $\Gamma(p, z)$ in the case of a compound Poisson input}

The Lagrange expansion technique used in this section is similar to that which is used in the derivation of the Borel-Tanner distribution in queueing theory, which is a special case.

Let the points of increase of $\xi(t)$ follow a Poisson law with parameter $\lambda$, and let the distribution function of the jumps be $B(x)$. Then

$$
K(t, x)=\sum_{n=0}^{\infty} e^{-\lambda t} \frac{(\lambda t)^{n}}{n !} B_{n}(x), \quad t \geqq 0,
$$

where $B_{n}(x)$ is the $n$-th convolution of $B(x)$ with itself. Expanding $e^{-\lambda t}$ in powers of $t$ and multiplying out the two series, we find that $K(t, x)$ admits the expansion

$$
K(t, x)=U(x)\left[1+t K_{10}(0, x)+\frac{t^{2}}{2 !} K_{20}(0, x)+\cdots\right]
$$

where $K_{n 0}(0, x)$ represents the $n$-th derivative of $K(t, x)$ with respect to the first argument, $t$, for $t=0$, and $U(x)$ is the Heaviside unit function, defined previously. The $K_{n 0}(0, x)$ are given by

It follows that

$$
K_{n 0}(0, x)=(-1)^{n} \lambda^{n} \sum_{k=0}^{n}(-1)^{k}\left(\begin{array}{l}
n \\
k
\end{array}\right) B_{k}(x) .
$$

so that

$$
\left|K_{n 0}(0, x)\right| \leqq \lambda^{n} \sum_{k=0}^{n}\left(\begin{array}{l}
n \\
k
\end{array}\right)=(2 \lambda)^{n},
$$

$$
\left|\sum_{n=0}^{N} \frac{t^{n}}{n !} K_{n 0}(0, x)\right| \leqq \sum_{n=0}^{N} \frac{(2 \lambda t)^{n}}{n !} \leqq e^{2 \lambda t}, \quad t \geqq 0 .
$$

Thus the partial sums of the expansion (14) are uniformly dominated by $e^{2 \lambda i}$. 
Theorem 4. If $\xi(t)$ is a Compound Poisson Process, the distribution function, $G(t, z)$, of $\tau(z)$ is given by the formula

$G(t, z)= \begin{cases}\frac{z}{t} K(t, t-z)-\int_{z}^{t} \frac{z}{u^{2}}\left[u K_{10}(u, u-z)-K(u, u-z)\right] d u & \text { if } t \geqq z, \\ 0 & \text { otherwise. }\end{cases}$

PRoof. Taking the Laplace-Stieltjes transform of (14) term by term, and equating the coefficients of the powers of $t$, we find that

$$
p \int_{0}^{\infty} e^{-p x} K_{n 0}(0, x) U(x) d x=(-1)^{n}[\alpha(p)]^{n}, n=0,1,2, \cdots .
$$

From this, we deduce, using the usual rules for change of variable in Laplace transforms,

$$
e^{-p z}[\alpha(p)]^{n}=(-1)^{n} p \int_{0}^{\infty} e^{-p t} K_{n 0}(0, t-z) U(t-z) d t,
$$

and, denoting the Laplace-Stieltjes transform of $f(t)$, (which can be written in the two equivalent forms $\int_{0}^{\infty} e^{-p t} d f(t), p \int_{0}^{\infty} e^{-p t} f(t) d t$,) by $\mathscr{L}[f(t)]$, we can write

$$
\begin{aligned}
\frac{1}{n !} \frac{d^{n-1}}{d p^{n-1}}\left[e^{-p t}\{\alpha(p)\}^{n}\right]= & -\frac{p}{n !} \int_{0}^{\infty} e^{-p t} t^{n-1} K_{n 0}(0, t-z) U(t-z) d t \\
& +\frac{n-1}{n !} \int_{0}^{\infty} e^{-p t} t^{n-2} K_{n 0}(0, t-z) U(t-z) d t, \\
= & -\frac{p}{n !} \int_{0}^{\infty} e^{-p t} t^{n-1} K_{n 0}(0, t-z) U(t-z) d t \\
& +\frac{n-1}{n !} p \int_{0}^{\infty} e^{-p t} d t \int_{0}^{\infty} u^{n-2} K_{n 0}(0, u-z) U(u-x) d u, \\
= & \mathscr{L}\left[\frac{t^{n-1}}{n !} K_{n 0}(0, t-z) U(t-z)\right. \\
& \left.+\frac{n-1}{n !} \int_{0}^{t} u^{n-2} K_{n 0}(0, u-z) C^{T}(u-z) d u\right] .
\end{aligned}
$$

We now use the inequalities

$$
\begin{aligned}
\left|\sum_{n=0}^{N} \frac{t^{n-1}}{n !} K_{n 0}(0, t-z)\right| & \leqq \frac{1}{t} e^{2 \lambda t} U(t-z), \\
\left|\sum_{n=0}^{N} \frac{n-1}{n !} \int_{0}^{t} u^{n-2} K_{n 0}(0, u-z) d u\right| & \leqq \frac{1}{t} e^{2 \lambda t} U(t-z),
\end{aligned}
$$

which follow easily from (15). It follows that the sums involved in the inequal- 
[10] On the distribution of the time to first emptiness of a store with stochastic input $\mathbf{5 1 5}$

ities are uniformly dominated by $(1 / t) e^{2 \lambda t} U(t-z)$, and this function in turn has a convergent Laplace-Stieltjes transform for all $p>2 \lambda, z>0$.

Using now Lebesgue's dominated convergence theorem, (see Loève [6], p. 125) we can sum equation (16) from $n=1$ to $n=+\infty$, and we obtain

$$
\begin{aligned}
-z \sum_{n=1}^{\infty} \frac{1}{n !} \frac{d^{n-1}}{d p^{n-1}}\left[e^{-p z}\{\alpha(p)\}^{n}\right] & =\mathscr{L}\left[\frac{z}{t}\{K(t, t-z)-1\} U(t-z)\right. \\
& -\int_{0}^{t} \frac{z}{u} K_{10}(u, u-z) U(u-z) d u \\
& \left.+\int_{0}^{t} \frac{z}{u^{2}}\{K(u, u-z)-1\} U(u-z) d u\right] .
\end{aligned}
$$

Replacing in (9), and using

$$
\begin{gathered}
e^{-p z}=p \int_{0}^{\infty} e^{-p t} U(t-z) d t, \\
\int_{0}^{t} \frac{z}{u^{2}} U(u-z) d u=\left(1-\frac{z}{t}\right) U(t-z),
\end{gathered}
$$

we finally find

$$
\begin{aligned}
\Gamma(p, z)=\mathscr{L}\left[\frac{z}{t} K(t, t-z) U(t-z)\right. & -\int_{0}^{t} \frac{z}{u} K_{10}(u, u-z) U(u-z) d u \\
& \left.+\int_{0}^{t} \frac{z}{u^{2}} K(u, u-z) U(u-z) d u\right] .
\end{aligned}
$$

But as the Lagrange expansion (9) holds for all $p$ such that $\operatorname{Re}(p)>0$, it follows from the uniqueness property of the Laplace-Stieltjes transform (see Widder [7], p. 63) that if $G(t, z)$ is the distribution function of $\tau(z)$, we have

$$
\begin{aligned}
G(t, z)=\frac{z}{t} K(t, t-z) U(t-z) & -\int_{0}^{t} \frac{z}{u} K_{10}(u, u-z) U(u-z) d u \\
& +\int_{0}^{t} \frac{z}{u^{2}} K(u, u-z) U(u-z) d u .
\end{aligned}
$$

This can be rewritten more simply

(17) $G(t, z) \begin{cases}\frac{z}{t} K(t, t-z)-\int_{z}^{t} \frac{z}{u^{2}}\left[\imath K_{10}(u, u-z)-K(\mathfrak{v}, u-z)\right] d u & \text { if } t \geqq z \\ 0 & \text { otherwise. }\end{cases}$

COROLLARY. If, for fixed $z, K(t, x)$ has continuons derivatives in both $t$ and $x$ at the point $(t, t-z)$, and if we write 


$$
\frac{\partial}{\partial x} K(t, x)=k(t, x)
$$

then at the point $(t, z), G(t, z)$ has a continuous partial derivative in $t$, given by

$$
\frac{\partial}{\partial t} G(t, z)=g(t, z)=\frac{z}{t} k(t, t-z) .
$$

Proof. Differentiating both sides of (17), we obtain

$$
\begin{aligned}
g(t, z)=\frac{\partial}{\partial t} G(t, z)= & -\frac{z}{t^{2}} K(t, t-z)+\frac{z}{t} K_{10}(t, t-z)+\frac{z}{t} k(t, t-z) \\
& -\frac{z}{t} K_{10}(t, t-z)+\frac{z}{t^{2}} K(t, t-z), \\
& =\frac{z}{t} k(t, t-z) .
\end{aligned}
$$

This is Kendall's formula.

\section{The case of a discrete input}

Let us now assume that the input $\xi(t)$ takes only integral values. It is then clear that emptiness can occur only at times $z+n$, where $n=0,12, \cdots$. We shall write

$$
\begin{aligned}
& P\{\xi(t)=n\}=p_{n}(t), \\
& P\{\tau(z)=z+n\}=q_{n}(z),
\end{aligned}
$$

and we shall assume that the $p_{n}(t)$ have continuous derivatives. We then have

$$
\begin{aligned}
K(t, x) & =\sum_{k=0}^{[x]} p_{k}(t), \\
G(t, z) & =\sum_{k=0}^{[t-z]} q_{k}(z) .
\end{aligned}
$$

Equation (17) now takes the form

$$
\sum_{k=0}^{n} q_{k}(z)=\frac{z}{z+n} \sum_{k=0}^{n} p_{k}(z+n)-\int_{z}^{z+n} \frac{z}{u^{2}}\left[u \sum_{k=0}^{[u-z]} p_{k}^{\prime}(u)-\sum_{k=0}^{[u-z]} p_{k}(u)\right] d u .
$$

Write $n-1$ for $n$ and subtract. We find

$$
\begin{aligned}
q_{n}(z)=\frac{z}{z+n} p_{n}(z+n) & +\sum_{k=0}^{n-1} z\left[\frac{p_{k}(z+n)}{z+n}-\frac{p_{k}(z+n-1)}{z+n-1}\right] \\
& -\int_{z+n-1}^{z+n} \frac{z}{u^{2}}\left[u \sum_{k=0}^{[u-z]} p_{k}^{\prime}(u)-\sum_{k=0}^{[u-z]} p_{k}(u)\right] d u .
\end{aligned}
$$


It is easily checked that the last two terms of the right-hand side of this equation cancel out, and we are left with

$$
q_{n}(z)=\frac{z}{z+n} p_{n}(z+n)
$$

which is precisely Lloyd's formula.

\section{References}

[1] Benes, V. E. (1957), On queues with Poisson arrivals, Ann. Math. Stat, 28, 670-677.

[2] Gnedenko, B. V. and Kolmogorov, A. N. (1954), Limit Distributions for Sums of Independent Random Variables, Addison-Wesley, Cambridge, Mass.

[3] Kingman, J. F. C. (1963), On continuous time models in the theory of dams, this Journal $3,480-87$.

[4] Kendall, D. G. (1957), Some problems in the theory of dams, J. R. Statist. Soc. B, 19, $207-212$.

[5] Lloyd, E. H. (1963), The epochs of emptiness of a semi-infinite discrete reservoir, $J . R$. Statist. Soc. B, 25, 131-136.

[6] Loève, M. (1960), Probability Theory, (2nd ed.) Van Nostrand, Princeton.

[7] Widder, D. V. (1941), The Laplace Transform, Princeton University Press.

University of Tasmania, Australia. 